\title{
A proof of Aronhold's theorem upon quartic curves
}

\author{
By H. W. Richmond.
}

(Received 21st April, 1940. Read 3rd May, 1940.)

It is to be expected that a finite number of plane curves of order four should have seven given lines as bitangents, because the number of conditions imposed is equal to the number of effective free constants in the equation of such a curve, viz., 14. Aronhold ${ }^{1}$ made the interesting discovery that one curve could be determined in which no three of the given lines have their six points of contact on a conic. The method, due to Geiser, of obtaining the bitangents as projections of the lines of a cubic surface ${ }^{2}$ leads to a simple proof of the existence of this quartic.

Let $2,3,4,5,6,7,8$ be seven lines in a plane. Take any point $V$ outside the plane, and in the planes which contain $V$ and $7, V$ and 8 , respectively, take any two lines $7^{\prime}, 8^{\prime}$, which do not pass through $V$ and do not intersect. 7 and 8 are thus projections from $V$ of two base-lines $7^{\prime}$ and $8^{\prime} ; 2,3,4, \tilde{0}, 6$ are obtained as projections of five transversals $2^{\prime}, 3^{\prime}, 4^{\prime}, 5^{\prime}, 6^{\prime}$, definite lines which intersect $7^{\prime}, 8^{\prime}$. The base-lines and transversals lie upon a pencil of cubic surfaces of which one member passes through $V$ : projecting from $V$, we have a plane quartic with the given lines as bitangents. Moreover in Schläfli's notation the base-lines may be taken as $a_{1}$ and $b_{1}$, and the transversals as $c_{12}, c_{13}, c_{14}, c_{15}, c_{16}$. By projection these become bitangents denoted by 17, 18 and 12, 13, 14, 15, 16 which (as Salmon points out) form an A ronhold set.

The tangent planes of all surfaces of the pencil at various points of $7^{\prime}$ pass through $7^{\prime}$ and coincide at the five points where $7^{\prime}$ is met by a transversal: this cannot be unless the tangent planes coincide at every point of $7^{\prime}$, a fact which is confirmed when we attempt to determine the complete intersection of the surfaces of the pencil. There are two points of $7^{\prime}$ whose tangent planes pass through $V$ and these points are projected into the points of contact of 7 with

1 Salmon, Higher Plane Curves, 3rd Edition (Dublin, 1879), 234-240.

2 Geiser, Math. Annalen, 1 (1869), 129. 
Aronhold's quartic. (They will be points of contact of a bitangent of a sextic curve if we project an arbitrary surface of the pencil.) Now the pencil includes two ruled cubic surfaces, one of them having $7^{\prime}$ as its double line. The lines on this surface are projected into tangents of a curve of class three; $7^{\prime}$ is projected into a double tangent, its points of contact being the same as those of Aronhold's quartic. This rule for determining the points of contact of his quartic is due to Aronhold. It will be observed that when (as here) we work with tangential coordinates, to make a given line a double tangent of a curve imposes three conditions; the plane curve of class three is therefore fully defined by the conditions imposed upon it.

King's College,

Cambridge. 